\title{
EPIDEMIOLOGIA DAS FRATURAS DE ACETÁBULO TRATADAS NO INSTITUTO NACIONAL DE TRAUMATOLOGIA E ORTOPEDIA (INTO)
}

\author{
EPIDEMIOLOGY OF ACETABULUM FRACTURES TREATED AT THE INSTITUTO \\ NACIONAL DE TRAUMATOLOGIA E ORTOPEDIA (INTO)
}

Marcus Vinícius Fernandes Dias', Flavio Goldszłajn², João Matheus Guimarães ${ }^{3}$, José Afraneo Grizendi4, Marcos Correia ${ }^{5}$, Tito Henrique Rocha ${ }^{6}$

\section{RESUMO}

Objetivos: O propósito do presente estudo é revisar aspectos epidemiológicos das fraturas desviadas do acetábulo tratadas cirurgicamente no Instituto Nacional de Ortopedia e Traumatologia (INTO). Métodos: Analisamos, retrospectivamente, 126 fraturas de acetábulo tratadas cirurgicamente no INTO, no período de março de 2006 a novembro de 2008. Levamos em consideração: idade; sexo; mecanismo de trauma; classificação da lesão; tempo decorrido entre o trauma e o tratamento cirúrgico; lado acometido; lesões ósseas associadas. Resultados: 76,8\% são do sexo masculino, a média da idade foi de 39,6 anos; quanto ao mecanismo de trauma, 59\% foram por acidente automobilístico; o tempo decorrido entre a lesão e o tratamento cirúrgico foi em média 16,4 dias; 55\% casos foram do lado direito; 30\% pacientes apresentavam fraturas associadas. Conclusão: A maior parte dos pacientes é do sexo masculino, na faixa etária economicamente ativa, e vítima de acidente de trânsito, sendo as fraturas da reborda e/ou coluna posterior as mais frequentes. Lesões associadas são comuns e a maior parte das fraturas operadas em nosso serviço é envelhecida.

Descritores - Acetábulo/cirurgia; Fraturas Ósseas; Epidemiologia; Ferimentos e Lesões

\section{ABSTRACT}

Objectives: The purpose of this study is to review epidemiological aspects of displacement fractures of the acetabulum treated surgically at the Instituto Nacional de Traumatologia e Ortopoedia (INTO). Methods: We retrospectively analyzed 126 acetabulum fractures treated surgically at the INTO in the period from March 2006 to November 2008. The following elements were taken in account: age, sex, mechanism of injury, classification, time elapsed between trauma and surgery, affected side, associated bone injuries. Results: $76.8 \%$ were male, the mean age was 39.6 years. $59 \%$ of the traumas were due to traffic accidents; time between injury and surgery was on average 16.4 days; $55 \%$ of cases were on the right side; $30 \%$ of patients had associated fractures. Conclusion: Most patients are male, in an economically active age group, and are victims of traffic accidents. Fractures of edge and/or posterior column are the most frequent. Associated injuries are common and most of the fractures operated in our service are delayed.

Keywords - Acetabulum/surgery; Fractures, Bone; Epidemiology; Wounds and Injuries

\section{INTRODUÇÃO}

Nos últimos 40 anos, o tratamento das fraturas desviadas do acetábulo passou de não-cirúrgico para uma abordagem preferencialmente cirúrgica. A partir da contribuição de Letournel ${ }^{(1)}$, a redução anatômica aberta, seguida de fixação interna rígida, passou a ser considerada como padrão ouro no tratamento das fraturas desviadas do acetábulo.

Antes do advento do automóvel e da motocicleta na vida moderna, esse tipo de lesão era causada essencialmente por forças em diferentes graus de intensidade em adução ou abdução do membro inferior, associada

\footnotetext{
1 - Médico Assistente do Centro de Pelve e Acetábulo do Instituto Nacional de Traumatologia e Ortopedia - INTO, Rio de Janeiro, Brasil.

2 - Chefe do Centro de Pelve e Acetábulo do Instituto Nacional de Traumatologia e Ortopedia - INTO, Rio de Janeiro, Brasil.

3 - Chefe do Serviço do Instituto Nacional de Traumatologia e Ortopedia - INTO, Rio de Janeiro, Brasil.

4 - Médico Assistente do Centro de Trauma do Instituto Nacional de Traumatologia e Ortopedia - INTO, Rio de Janeiro, Brasil

5 - Chefe do Centro de Trauma do Idoso do Instituto Nacional de Traumatologia e Ortopedia - INTO, Rio de Janeiro, Brasil.

6 - Chefe da Coordenação de Desenvolvimento Institucional do Instituto Nacional de Traumatologia e Ortopedia - INTO, Rio de Janeiro, Brasil.
}

Trabalho realizado no Centro de Pelve e Acetábulo do Instituto Nacional de Traumatologia e Ortopedia do Ministério da Saúde (INTO-MS), RJ, Brasil. Correspondência: Rua Presidente Backer, 25/902, Icaraí - 24220-045 - Niterói, RJ. E-mail: mvortopedia@hotmail.com 
a alguma flexão em rotação interna ou externa. Atualmente, o trauma de alta energia cinética, usualmente relacionado ao trânsito, predomina como etiologia ${ }^{(2)}$.

Cerca de 2/3 dos pacientes são jovens e lesões associadas são comumente encontradas nesse grupo, podendo variar entre $40 \%$ a $75 \%{ }^{(3)}$.

A importância do pronto reconhecimento desse grupo de fraturas, bem como o estabelecimento o quanto antes do tratamento definitivo, são frequentemente ressaltados na literatura. E, a fim de otimizar o tratamento dessas lesões, faz-se mister a compreensão da personalidade da fratura e, nesse sentido, preconiza-se a utilização de algumas classificações que apontam o prognóstico e o tratamento ${ }^{(4)}$.

Ainda nesse sentido, o tempo decorrido entre a lesão e a instituição do tratamento definitivo é considerado como de suma importância na obtenção de uma redução anatômica, a qual se torna mais difícil à medida que esse tratamento é adiado e, por conseguinte, acaba influenciando o resultado funcional desses pacientes ${ }^{(5)}$. Diante da constante evolução na abordagem dessas lesões, bem como a morbidade associada às mesmas, suscitando frequente debate sobre o tema, motivamo-nos a estudar as características desse grupo de fraturas.

O propósito do presente estudo é revisar aspectos epidemiológicos das fraturas desviadas do acetábulo tratadas cirurgicamente no Instituto Nacional de Ortopedia e Traumatologia (INTO), comparando os nossos resultados com os achados da literatura.

\section{METODOLOGIA}

Foram analisadas, de forma retrospectiva, 126 fraturas de acetábulo tratadas cirurgicamente por médicos do Centro de Pelve e Acetábulo do INTO, no período compreendido entre março de 2006 e novembro de 2008. O levantamento foi realizado por meio de busca ativa no banco de dados do INTO por meio do Código Internacional de Doenças (CID) S324, que corresponde às fraturas do acetábulo. Procedeu-se então, a partir do número de registro do paciente no INTO, à consulta ao prontuário e aos arquivos de imagens dos respectivos pacientes. Os critérios de inclusão foram: pacientes submetidos ao tratamento cirúrgico de fraturas do acetábulo, independente de possuírem ou não lesões associadas; de ambos os sexos; de todas as idades; e que realizaram o seguimento ambulatorial até fevereiro de 2009. Foram excluídos: os pacientes tratados por método não-cirúrgico, os que perderam o seguimento ambulatorial e os que possuíam prontuário e/ou exames de imagem contendo deficiência nos dados levados em conta em nosso trabalho. Foram levados em consideração: idade; sexo; mecanismo de trauma; classificação da lesão segundo Tile ${ }^{(6)}$, avaliada por três cirurgiões familiarizados com o tratamento das fraturas do acetábulo, após análise de radiografias em ânteroposterior da bacia, alar e obturatriz e tomografia com corte de $1,8 \mathrm{~mm}$ com reconstrução nos três planos (coronal, axial e sagital); tempo decorrido entre o trauma e o tratamento cirúrgico; lado acometido; lesões ósseas associadas. Os resultados foram avaliados por média e desvio padrão.

Dos 126 pacientes analisados, 53 foram excluídos da análise, sendo sete por método não-cirúrgico como tratamento de escolha, nove por perda de seguimento e 37 por apresentarem prontuário e/ou exames de imagens com informações incompletas em relação aos quesitos levados em consideração no presente trabalho. Tal exclusão se deu em virtude de falha em obter informações referentes ao tempo de evolução da fratura na ocasião do tratamento cirúrgico e em relação ao mecanismo de trauma da lesão. Acreditamos que, embora uma parte substancial de nossa casuística tenha sido excluída, tal fato não trouxe consigo alterações significativas no perfil epidemiológico do nosso trabalho, uma vez que houve predomínio das lesões envelhecidas, que é uma característica global em nossa instituição, e do acidente automobilístico como mecanismo de trauma. Dos 126 analisados, apenas 73 foram efetivamente incluídos em nossa análise.

O presente trabalho encontra-se em conformidade com a Declaração de Helsinki da Associação Médica Mundial.

\section{RESULTADOS}

Dos 73 pacientes, $56(76,8 \%)$ são do sexo masculino e $17(23,2 \%)$ do feminino (Tabela 1); a média da idade foi de 39,6 $\pm 3,8$ anos (mínima de 12 e máxima de 89 anos) (Tabela 2); quanto ao mecanismo de trauma, 43 (59\%) foram por acidente automobilístico, 14 (19\%) por acidente envolvendo motocicleta, seis $(8,2 \%)$ atropelamentos, nove $(12,4 \%)$ quedas, um $(1,4 \%)$ acidente naval (Tabela 3); segundo a classificação de Tile ${ }^{(6)}: 20$

Tabela 1 - Distribuição por sexo.

\begin{tabular}{c|c|c}
\hline Sexo Masculino & Feminino & \\
\hline Casos & 56 & 17 \\
\hline$\%$ & 76,8 & 23,2 \\
\hline
\end{tabular}


Tabela 2 - Distribuição por idade em anos.

\begin{tabular}{c|c|c|c}
\hline Idade & Mínima & Máxima & Média \pm desvio padrão \\
\hline Anos & 12 & 89 & $39,6 \pm 3,8$ \\
\hline
\end{tabular}

Tabela 3 - Mecanismo de trauma.

\begin{tabular}{c|c|c|c|c|c}
\hline Mecanismo & Automóvel & Motocicleta & Atropelamento & Queda & Naval \\
\hline Casos & 43 & 14 & 6 & 9 & 1 \\
\hline$\%$ & 59 & 19 & 8,2 & 12,4 & 1,4 \\
\hline
\end{tabular}

$(27,4 \%)$ casos podem ser classificados como A1, sete (9,6\%) como A2, dois (2,7\%) como A3, 25 (34,4\%) como B1, seis $(8,2 \%)$ como B2, $11(15 \%)$ como $\mathrm{C} 1$, dois $(2,7 \%)$ como $\mathrm{C} 2$, e não foram encontradas lesões classificadas como B3 e C3. Não houve discordância entre os observadores em nenhum dos casos quanto ao tipo de fratura (Tabela 4); o tempo decorrido entre a lesão e o tratamento cirúrgico foi, em média, de $16,4 \pm 1,1$ dias (mínimo de um e máximo de 64 dias) (Tabela 5); $40(55 \%)$ casos foram do lado direito e 33 (45\%) do lado esquerdo (Tabela 6); 22 (30\%) pacientes apresentavam fraturas associadas, sendo oito $(36,3 \%)$ lesões do anel pélvico, cinco $(22,5 \%)$ de fêmur, quatro $(17,2 \%)$ de tíbia, duas de punho (9\%), uma (5\%) de patela, uma (5\%) de clavícula, e uma (5\%) de anel pélvico, fêmur e tíbia (Tabela 7).

Tabela 4 - Distribuição pela classificação de Tile ${ }^{(6)}$.

\begin{tabular}{c|c|c|c|c|c|c|c|c|c}
\hline Tipo & A1 & A2 & A3 & B1 & B2 & B3 & C1 & C2 & C3 \\
\hline Casos & 20 & 7 & 2 & 25 & 6 & XXX & 11 & 2 & XXX \\
\hline$\%$ & 27,4 & 9,6 & 2,7 & 34,4 & 8,2 & XXX & 15 & 2,7 & XXX \\
\hline
\end{tabular}

Tabela 5 - Intervalo de tempo entre a fratura e o tratamento cirúrgico.

\begin{tabular}{c|c|c|c}
\hline $\begin{array}{c}\text { Tempo } \\
\text { (dias) }\end{array}$ & Mínimo & Máximo & Média \pm desvio padrão \\
\hline Casos & 1 & 64 & $16,4 \pm 1,1$ \\
\hline
\end{tabular}

Tabela 6 - Lado acometido.

\begin{tabular}{c|c|c}
\hline Lado acometido & Esquerdo & Direito \\
\hline Casos & 33 & 40 \\
\hline$\%$ & 45 & 55 \\
\hline
\end{tabular}

Tabela 7 - Lesões ósseas associadas.

\section{DISCUSSÃO}

Letournel $^{(1)}$ revolucionou o tratamento das fraturas do acetábulo. Juntos, desenvolveram uma melhor compreensão deste tipo de lesão, racionalizando a abordagem cirúrgica. A partir desses conceitos, diversos cirurgiões se encorajaram a tratar tais lesões de forma cirúrgica e, desde então, inúmeros trabalhos acerca do tratamento cirúrgico das lesões acetabulares foram publicados.

Em extensa revisão publicada em 2005, Giannoudis et $a l^{(7)}$ analisaram 160 trabalhos sobre fraturas desviadas de acetábulo e, destes, apenas 34 preenchiam o seu rigoroso critério de inclusão, totalizando 3.670 fraturas desviadas em 3.669 pacientes.

Quanto ao sexo, Giannoudis et al ${ }^{(7)}$ encontraram $69,4 \%$ do sexo masculino. Tal achado é corroborado pela nossa casuística, em que a grande maioria é do sexo masculino (76,8\%). Sua média de idade foi de $38,6 \pm 4,6$ anos, a nossa foi de 39,6 anos $\pm 3,8$, fato que ressalta a maior prevalência na população jovem e economicamente ativa. $\mathrm{O}$ acidente de trânsito foi responsável por $80,5 \%$ dos casos analisados por Giannoudis et $a \mathbf{l}^{(7)}$. Em nossa estatística, a mesma causa foi encontrada em $78 \%$ dos casos. Giannoudis et al ${ }^{(7)}$ levaram em consideração a classificação de Letournel ${ }^{(1)}$ para fins de análise. Essa classificação é a encontrada na maioria dos artigos. Em nossos casos utilizamos a classificação de Tile ${ }^{(6)}$ por ser a de escolha em nosso serviço. No entanto, a diferença na opção da classificação não nos impede de realizar uma comparação dos resultados. No trabalho de Giannoudis et $a l^{(7)}$, a maior incidência foi das fraturas da coluna posterior $(23,9 \%)$, seguida pelas fraturas das duas colunas $(22 \%)$. Nossos resultados apontam para um maior acometimento da reborda e/ou coluna posterior, totalizando $37 \%$. A fratura da dupla coluna, ao contrário do trabalho de Giannoudis et $a l^{(7)}$, foi encontrada em apenas $13,7 \%$ dos casos. Matos et al ${ }^{(8)}$ avaliaram a reprodutibilidade da classificação de Tile ${ }^{(6)}$ e a recomendaram para o uso na prática clínica, fato que ratifica o uso de tal classificação em nosso serviço. Em contrapartida, Sancineto et $a l^{(9)}$ encontraram baixa reprodutibilidade inter e intrapessoal da classificação de Letournel $^{(1)}$. A média de tempo entre a lesão e a cirurgia foi de 8,9 2,9 dias na análise de Giannoudis et $a l^{(7)}$.Ao contrário, em nossa experiência, prevaleceram as lesões

\begin{tabular}{|c|c|c|c|c|c|c|c|c|}
\hline Lesões associadas & Pelve & Fêmur & Tíbia & Punho & Patela & Clavícula & Pelve/ fêmur/ tíbia & Total \\
\hline Casos & 8 & 5 & 4 & 2 & 1 & 1 & 1 & 22 \\
\hline$\%$ & 36,3 & 22,5 & 17,2 & 9 & 5 & 5 & 5 & 100 \\
\hline
\end{tabular}


envelhecidas com uma média de 16,4 $\pm 1,1$ dias. Matta et $a l^{(3)}$ relatam que fraturas com 14 ou mais dias de evolução já têm a qualidade da redução comprometida pela dificuldade cirúrgica, considerando-as, portanto, como envelhecidas. Em nosso meio, Köberle et al ${ }^{(10)}$ obtiveram resultados variando de zero a 150 dias, sendo 25 operados antes de 21 dias e 17 após três semanas, de um total de 42 casos. Tal fato reflete, em parte, a dificuldade de acesso ao tratamento de tais lesões em nosso meio. No que tange ao lado acometido, houve um predomínio do acometimento do lado direito sobre o esquerdo, $55 \%$ e $45 \%$, respectivamente. Resultado inverso foi encontrado por Knop et al ${ }^{(11)}$. Esses autores publicaram em uma casuística de 40 casos, 16 (40\%) do lado direito, 22 do lado esquerdo (55\%) e dois (5\%) casos de acometimento bilateral. Giannoudis et $a l^{(7)}$ não levaram em conta tal informação em sua meta-análise e outros trabalhos consultados não mencionam o fato ${ }^{(12-14)}$, o que nos faz supor que tal informação é omitida por não trazer em si maiores repercussões do ponto de vista de tratamento e prognóstico.

Lesões associadas em pacientes com fratura acetabular são fartamente documentadas, sendo fraturas dos membros e traumatismo craniencefálico as mais comuns ${ }^{(10,12-14)}$. Outras fraturas associadas perfazem um total de $45 \%$, segundo Giannoudis et $a l^{(7)}$, contrastando com $30 \%$ dessa associação em nossa casuística. Também em contraste com os nossos resultados, esses autores encontraram uma predominância de fraturas nos membros em relação às fraturas do anel pélvico, resultado oposto ao nosso. Tal diferença não pôde ser explicada pelos autores. Em razão de nosso serviço não possuir emergência aberta e ser um hospital exclusivamente ortopédico, não levamos em consideração lesões associadas em outros sistemas que não o musculoesquelético em virtude do perfil de nossa instituição e dos pacientes que nela são assistidos.

\section{REFERÊNCIAS}

1. Letournel E. Acetabulum fractures: classification and management. Clin Orthop Relat Res. 1980;(151):81-106.

2. Alonso JE, Volgas DA, Giordano V, Stannard JP. A review of the treatment of hip dislocations associated with acetabular fractures. Clin Orthop Relat Res. 2000;(377):32-43

3. Matta JM, Anderson LM, Epstein HC, Hendricks P. Fractures of the acetabulum. A retrospective analysis. Clin Orthop Relat Res. 1986;(205):230-40.

4. Balbachevsky D, Pires RE, Faloppa F, Reis F. Tratamento das fraturas da pelve e acetábulo pela via de Stoppa modificada. Acta Ortop Bras. 2006;14(4):190-2.

5. Liebergall M, Mosheiff R, Low J, Goldvirt M, Matan Y, Segal D. Acetabular fractures. Clinical outcome of surgical treatment. Clin Orthop Relat Res. 1999;(366):205-16.

6. Tile M. Fractures of the acetabulum. Orthop Clin North Am. 1980;11(3):481-506.

7. Giannoudis PV, Grotz MR, Papakostidis C, Dinopoulos H. Operative treatment of displaced fractures of the acetabulum. A meta-analysis. J Bone Joint Surg Br. 2005;87(1):2-9.
Dessa forma, o presente estudo chama a atenção para o fato de apesar de possuirmos em nossa instituição um centro que se dedica exclusivamente às fraturas de anel pélvico e de acetábulo, ainda assim temos dificuldade em proceder o tratamento cirúrgico em um tempo ideal como preconizado $^{(3)}$. Isso reflete as dificuldades na rede de saúde local em prestar o atendimento inicial, reconhecer a natureza cirúrgica da lesão de forma imediata e estabelecer contato com a central reguladora a fim de viabilizar a pronta transferência desses pacientes para um centro especializado. E esse atraso na instituição do tratamento definitivo adequado, naturalmente, reflete-se no resultado clínico radiológico desse grupo de pacientes ${ }^{(3,5,7)}$.

A repercussão clínica dessas lesões foge ao escopo desse trabalho; no entanto, motivados por esse estudo, pretendemos correlacionar nossos achados epidemiológicos com a abordagem terapêutica instituída e sua repercussão funcional nos pacientes submetidos à osteossíntese das fraturas do acetábulo em nossa instituição em estudo posterior.

\section{CONCLUSÕES}

A maior parte dos pacientes é do sexo masculino, na faixa etária economicamente ativa, e vítima de acidente de trânsito, sendo que o acometimento da reborda e/ou coluna posterior ocorreu na maior parte dos casos, em consonância com a literatura.

Lesões associadas são frequentes e a maior parte das fraturas operadas em nosso serviço, ao contrário da literatura, é envelhecida.

\section{AGRADECIMENTO}

Ao Dr. Peter Giannoudis, da Universidade de Leeds no Reino Unido, pelas sugestões e incentivo na publicação desse trabalho.

8. Matos MA, Viveiros AM, Barreto BG, Pires RF. Reprodutibilidade da classificação de Tile para fraturas do acetábulo. Acta Ortop Bras. 2006:14(5):253-55.

9. Sancineto C, Barla J, Slullitel G, Della Valle AG, Comba F, Bongiovanni S et al. Reproductibilidad de la clasificacion de Letournel para las fracturas cetabulares: estudio sobre radiografias y tomografia computadorizada tridimensional. Rev Asoc Argent Ortop Traumatol. 2004;69(1):59-63.

10. Köberle G, Miranda JB, Yamanaka E, Grava ALS, Belangero WD. Fraturas do acetábulo: estudo dos resultados clínicos do tratamento cirúrgico precoce e tardio. Rev Bras Ortop. 1993;28(6):361-6.

11. Knop T, Silva LHP, Laghi R. Fraturas acetabulares: resultados de tratamento cirúrgico. Rev Bras Ortop. 1996;31(10):825-30.

12. Aho AJ, Isberg UK, Katevuo VK. Acetabular posterior wall fracture. 38 cases followed for 5 years. Acta Orthop Scand. 1986;57(2):101-5.

13. Chiu FY, Chen CM, Lo WH. Surgical treatment of displaced acetabular fractures 72 cases followed for 10 (6-14) years. Injury. 2000;31(3):181-5.

14. Deo SD, Tavares SP, Pandey RK, El-Saied G, Willett KM, Worlock PH. Operative management of acetabular fractures in Oxford. Injury. 2001;32(7):581-6. 